\title{
Apnea In A Term Neonate: Expanding The Differential Diagnosis
}

ISSN: 2576-9200

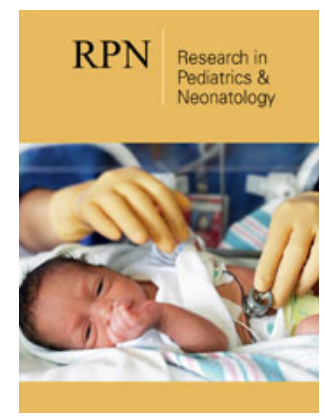

*Corresponding author: Audrey Miller,Department of Pediatrics, Division of Neonatology, West Virginia University School of Medicine, USA

Submission: 酸 November 10, 2020

Published: 制November 24, 2020

Volume 5 - Issue 2

How to cite this article: Audrey Miller, Rebecca Burke,Alfred Gest. Apnea In A Term Neonate: Expanding The Differential Diagnosis. Research in Pediatrics \& Neonatology. 5(2). RPN. 000606. 2020. DOI: 10.31031/RPN.2020.05.000606

Copyright@ Audrey Miller. This article is distributed under the terms of the Creative Commons Attribution 4.0 International License, which permits unrestricted use and redistribution provided that the original author and source are credited.

\author{
Audrey Miller ${ }^{1 *}$, Rebecca Burke ${ }^{1,2}$ and Alfred Gest ${ }^{1}$ \\ ${ }^{1}$ Department of Pediatrics, Division of Neonatology, West Virginia University School of \\ Medicine, USA \\ ${ }^{2}$ Department of Pediatrics, Division of Medical Genetics, West Virginia University School of \\ Medicine, USA
}

\author{
Abstract \\ Keywords:Hypoventilation;Apnea
}

Abbreviation Persistent Pulmonary Hypertension of Newborn (PPHN);Coarctation of Aorta (CoA);Patent Ductus of Arteriosus (PDA).

\section{Case Presentation}

A zero-day old female infant is admitted to the NICU for persistent oxygen desaturations and cyanosis. She was born at a gestational age of 39 weeks to a 31-year-old Gravida 4 Para 4 mother by spontaneous vaginal delivery. The pregnancy was uncomplicated. Maternal blood type was 0 positive and the remainder of the maternal serologies are unremarkable. Delivery was complicated by the presence of meconium stained fluid and a tight nuchal cord. APGAR scores were six at one minute and eight at five minutes. The infant required administration of CPAP in the delivery room for an oxygen saturation of seventy percent at five minutes of life. Following admission to the NICU, the infant had an escalating oxygen requirement and recurrent apneic events resulting in endotracheal intubation and mechanical ventilation.

The infant's birth weight is $3.310 \mathrm{~kg}\left(56^{\text {th }}\right.$ percentile on WHO Girls Curve), birth length is $49.5 \mathrm{~cm}\left(58^{\text {th }}\right.$ percentile on WHO Girls Curve $)$, and FOC is $35.6 \mathrm{~cm}\left(92^{\text {nd }}\right.$ percentile on WHO Girls Curve). Vital signs are significant for an axillary temperature of 36.8 degrees Celsius, heart rate of $132 \mathrm{bpm}$, blood pressure of $88 / 62$, and oxygen saturation of $97 \%$ on a fraction of inspired oxygen of $40 \%$. Her physical exam is remarkable for easy respiratory effort on mechanical ventilation with clear breath sounds and equal air entry. Her respiratory rate is slow for a neonate at 20 breaths per minute. She does not have a murmur. Her capillary refill is brisk and she has a normal heart rhythm on the monitor. She has normal muscle tone and patellar reflexes. She did not have any striking dysmorphic craniofacial features and her abdominal, genitourinary, musculoskeletal, and skin exams are grossly normal. She continues to periods of apnea while on mechanical ventilation. A chest radiograph demonstrates clear lung fields and a normal cardiac silhouette. The family history is significant for three older siblings who are healthy and developmentally typical.

Initial laboratory studies revealed a glucose of 77, a CBC with a WBC of 13.8 , hemoglobin of 19.6, hematocrit of 58, platelet count of 221, with a normal cell differential. Her venous blood gas revealed a pH of 7.22, $\mathrm{CO} 2$ of $64, \mathrm{P} 02$ of 41 , bicarbonate of 26 , and a base deficit of 3.6. Electrolyte and hepatic function panel revealed normal results. A lumbar puncture is performed and CSF analysis is normal. Blood and CSF cultures are negative. Herpes simplex virus studies are negative. A serum ammonia level is $85 \mathrm{umol} / \mathrm{L}$, which is within normal range for the hospital laboratory. A meconium drug screen is negative. Her state newborn screen is normal. A karyotype is performed which reveals a normal female chromosome complement, 46, XX. Her head ultrasound reveals no abnormality. A Brain MRI with and without contrast reveals a few punctate foci of restricted diffusion in the right hemisphere, with otherwise 
normal morphology and anatomy. Pediatric ENT is consulted for a flexible nasal endoscopy which demonstrates normal anatomy of the nares and upper airway. She has a normal electroencephalogram (EEG) for age without evidence of seizures. A medical genetics consultation is requested, and a chromosome microarray with SNP analysis, PHOX2B sequence and PARM analysis, and congenital myasthenic syndrome gene sequencing panel are sent.

\section{Discussion}

The differential diagnosis for a term infant with apnea is broad and varies based on age and pathological mechanism. Central apnea may be secondary to infectious etiologies (sepsis, meningitis, and encephalitis), seizures, birth trauma (intracranial hemorrhage, or hypoxic-ischemic encephalopathy), primary CNS malformations, metabolic derangements (acidosis, hypoglycemia, hyponatremia, hypernatremia, and inborn errors of metabolism), primary pulmonary disorders (pneumonia, pulmonary hemorrhage), congenital heart disease and intrauterine exposure to illicit drugs or other medications [1,2]. Upper airway malformations (choanal atresia, Pierre-Robin Sequence) and disorders associated with hypotonia are more likely to lead to obstructive apnea in term infants. Genetic disorders that lead to apnea and sleep-related hypoventilation in a term neonate include disorders presenting with central apnea such as congenital central hypoventilation syndrome (CCHS) and Joubert syndrome, as well as disorders leading to hypotonia and obstructive apnea such as Down syndrome, PraderWilli syndrome and congenital myotonic dystrophy [1,2].

The patient presented in this case had an abnormal PHOX2B sequence analysis result, consistent with a molecular diagnosis of congenital central hypoventilation syndrome. She was found to be heterozygous for a 26 polyalanine repeat expansion (PARM) in the PHOX2B gene. PHOX2B encodes a homeodomain transcription factor involved in neural crest and autonomic nervous system development [3]. Changes in neural crest cells in the brainstem alter chemoreceptor sensitivity to hypercapnia and hypoxia [3-5]. This leads to impaired breathing, particularly during non-REM sleep when breathing is dependent on autonomic control. Congenital central hypoventilation syndrome is a rare disorder of respiratory control and the autonomic nervous system and is thought to affect at least 1,000 individuals worldwide [3,4]. The condition results from a heterozygous molecular variant or polyalanine repeat expansion of the polyalanine repeat region located in the PHOX2B gene on chromosome 4 p12 in 92\% of affected individuals. The remaining individuals have a heterozygous variant in a non-polyalanine repeat mutation (NPARM) region of PHOX2B [3-4]. Significantly, there is a correlation between genotype and phenotype in this condition. Length of the PARM and the location of the variant in a NPARM region of PHOX2B predict phenotype and are used to guide management and disease surveillance [3,4].

Individuals with CCHS present with hypoventilation, shallow breathing, and decreased respiratory rates, especially during nonREM sleep [3,6]. Central apnea and cyanosis often result in the need for mechanical ventilation shortly after birth. Often these patients will not show signs of respiratory distress and will not increase their respiratory rate or tidal breathing in response to hypercapnia and hypoxia. Hirschsprung disease occurs in about $15-20 \%$ of patients with CCHS and is often associated with NPARMs [3]. Tumors of neural crest origin can also occur, also more common in patients with NPARMs [3]. Patients with CCHS frequently have autonomic nervous system dysfunction, including pupillary anomalies, blood pressure changes, temperature instability, and esophageal dysmotility as well as cardiac arrhythmias and prolonged sinus pauses [6]. Almost all individuals affected with CCHS require tracheostomy with long-term positive-pressure ventilation $[3,7]$. Diaphragm pacing may be an option to allow for tracheostomy decannulation in patients that are only ventilator-dependent during sleep [7]. Some patients require a cardiac pacemaker if they have prolonged sinus pauses. Respiratory stimulants, such as caffeine are often ineffective.

\section{Patient Course}

During her first few weeks of life, the patient failed multiple extubation attempts. Her apneic events did seem to follow a predictable course over the day, with more events occurring during light sleep. A trial of caffeine was conducted over three days, which did not result in decreased frequency of apnea. She had episodes of emesis and abdominal distention with feeds, however there was no clinical evidence for Hirschsprung's disease. She did have multiple episodes of temperature instability consistent with autonomic nervous system dysfunction which is well described in CCHS. At about one month of age, she developed EEG confirmed seizures, and was started on an antiepileptic. No cardiac arrhythmias occurred during the NICU hospitalization and 72-hour Holter testing was normal. Following identification of her PARM in PHOX2B, she underwent tracheostomy placement and she was discharged home on a ventilator. Both of her parents underwent testing for the familial polyalanine repeat expansion in PHOX2B and were found to be negative.

\section{References}

1. Kasi AS, Perez IA, Kun SS, Keens TG (2016) Congenital central hypoventilation syndrome: Diagnostic and management challenges. Pediatric Health Med Ther 7: 99-107.

2. Bishara J, Keens TG, Perez IA (2018) The genetics of congenital central hypoventilation syndrome: Clinical implications. The Application of Clinical Genetics 11: 135-144.

3. Weese-Mayer DE, Marazita ML, Rand CM, Berry-Kravis EM (2004) Congenital central hypoventilation syndrome, In: Adam MP, Ardinger HH, Pagon RA, (Eds.), Gene Reviews, University of Washington, Seattle, USA, pp. 1993-2020.

4. Weese-Mayer DE, Berry-Kravis EM, Ceccherini I, Keens TG, Loghmanee DA, et al. (2010) An official ATS clinical policy statement: Congenital central hypoventilation syndrome: Genetic basis, diagnosis, and management. Am J Respir Crit Care Med 181(6): 626-644.

5. Fisher M, Smeiles C, Jnah AJ, Ruiz ME, Difiore T, et al. (2019) Congenital central hypoventilation syndrome: A case-based learning opportunity for neonatal clinicians. Neonatal Netw 38(4): 217-225. 
6. Bardanzellu F, Pintus MC, Fanos V, Marcialis MA (2019) Neonatal congenital central hypoventilation syndrome: Why we should not sleep on it. Literature Review of forty-two neonatal onset cases. Curr Pediatr Rev 15(3): 139-153.
7. Zaidi S, Gandhi J, Vatsia S, Smith NL, Khan SA (2018) Congenital central hypoventilation syndrome: An overview of etiopathogenesis, associated pathologies, clinical presentation, and management. Auton Neurosci 210: 1-9. 